\title{
Análise do indicador de extração dentária a partir do contexto municipal
}

Analysis of the dental extraction index in a municipal context

Análisis del indicador de extracción dental a partir del contexto municipal

$\begin{array}{ll}\text { Recebido: } & 10 / 03 / 2017 \\ \text { Aprovado: } & 15 / 09 / 2017 \\ \text { Publicado: } & 05 / 04 / 2018\end{array}$

\section{Danielle Ramalho Barbosa da Silva ${ }^{1}$ Carolina Dantas Rocha Xavier de Lucena ${ }^{2}$ Danilson Ferreira da $\mathrm{Cruz}^{3}$ Nilcema Figueiredo 4 Paulo Savio Angeiras de Goes ${ }^{5}$ Edson Hilan Gomes de Lucena ${ }^{6}$}

Este estudo tem como objetivo analisar o indicador de exodontia em relação às variáveis de contexto dos municípios. Realizou-se um estudo transversal e ecológico. Por meio do DATASUS, coletaram-se dados do indicador de produção odontológica (Razão do Número de Exodontias sobre Procedimentos Odontológicos Básicos Individuais) referentes ao ano de 2016, de todos municípios brasileiros. Estes dados foram relacionados a Regiões do Brasil, Proporção de cobertura populacional estimada de saúde bucal na Estratégia Saúde da Família, número de Centros de Especialidades Odontológicas (CEO); Índice de Desenvolvimento Humano Municipal (IDHM) e coeficiente de Gini (ou índice de Gini). A região nordeste possui um número maior de municípios com resultados entre $12,1 \%$ e $25 \%$, quando comparada às demais regiões. As Regiões Norte e Nordeste apresentaram o maior percentual de municípios que realizam mais extrações dentárias, assim como aqueles que apresentam menor cobertura de Saúde Bucal. Não possuem CEO, têm IDHM muito baixo e índice GINI maior do que a média nacional. Conclui-se a necessidade de se inverter a oferta dos procedimentos odontológicos e garantir para a população, principalmente a menos favorecida socioeconomicamente, atendimentos e serviços que realizem ações de prevenção, manutenção e recuperação da saúde bucal, para que se possa reverter a perda dentária.

Descritores: Saúde bucal; Indicadores básicos de saúde; Extração dentária.

This study aims to evaluate the dental extraction index considering the many contextual variables of municipalities. This is a cross-sectional and ecological study. Data on odontological production (the number of Dental Extractions relative to the number of Individual Basic Odontological Procedures) in the year 2016, from all Brazilian municipalities, was found through DATASUS. These data were related to Brazilian Regions, Proportion of estimated populational coverage regarding oral health in the Family Health Strategy, number of Centers of Odontological Specialties (CEOs); Municipal Human Development Index (M-HDI) and the Gini coefficient (or Gini index). From the 12,1-2\%, the Northeast region has a superior index when compared to the other regions. The north and northeast regions show the highest percentage of cities that conduct the highest number of dental extractions, as well as those who present the smallest Oral Health coverage, do not have CEOs, have a low M-HDI, and a Gini index lower than the national average. Therefore, it becomes necessary to Invert the offer of odontological procedures and guarntee to the population, especially for those in difficult socioeconomic conditions, health care and services that can carry out preventive actions to maintain and recover oral health, so that dental loss can be avoided.

Descriptors: Oral health; Health status indicators; Tooth extraction.

Este estudio tiene como objetivo analizar el indicador de exodoncia en relación a las variables de contexto de los municipios. Se realizó un estudio transversal y ecológico. Por medio del DATASUS, se colectaron datos del indicador de producción odontológica (Razón del Número de Exodoncias sobre Procedimientos Odontológicos Básicos Individuales), referentes al año 2016, de todos los municipios brasileros. Estos datos fueron relacionados a Regiones de Brasil; proporción de cobertura poblacional estimada de salud bucal en la Estrategia Salud de la Familia; número de Centros de Especialidades Odontológicas (CEO); índice de Desarrollo Humano Municipal (IDHM) y coeficiente de GINI (o índice de GINI). De los 12,1-25\%, la región Noreste posee un índice superior al compararlo a las demás regiones. Las regiones Norte y Noreste presentaron el mayor porcentaje de municipios que realizan extracciones dentales, así como aquellos que presentan menor cobertura de Salud Bucal, no poseen CEO, tienen IDHM muy bajo e índice GINI mayor que el promedio nacional. Se concluye la necesidad de invertir en la oferta de los procedimientos odontológicos y garantizar para la población, principalmente la menos favorecida socioeconómicamente, atención y servicios que realicen acciones de prevención, manutención y recuperación de la salud bucal, para que se pueda revertir la pérdida dental. Descriptores: Salud bucal; Indicadores de salud; Extracción dental;

\footnotetext{
1. Graduanda do curso de Odontologia da Universidade Federal de Pernambuco (UFPE), Recife, PE, Brasil. ORCID: 0000-0001-7281-3439 E-mail:danielle.ramalho22@gmail.com

2. Cirurgiã Dentista. Especialista em Saúde da Família. Especialista em Epidemiologia. Mestre em Saúde Coletiva. Professora Substituta da UFPE, Recife, PE, Brasil. ORCID: 0000-0002-4652-9933 E-mail: krolrocha@yahoo.com.br

3. Cirurgiã Dentista. Especialista em Gestão do Cuidado em Saúde. Mestre em Modelos de Decisão e Saúde. Professor Assistente da UFPE, Recife, PE, Brasil. ORCID: 0000-0002-1948-3650 E-mail: danilsoncruz@yahoo.com.br

4. Cirurgiã Dentista. Especialista em Saúde Coletiva. Mestre e Doutora em Odontologia em Saúde Coletiva. Professora Associada da UFPE, Recife, PE, Brasil. ORCID: 0000-0001-6181-8728 E-mail: nilcema@gmail.com

5. Cirurgião Dentista. Mestre em Odontologia. Doutor em Epidemiologia e Saúde Pública. Professor Adjunto da UFPE, Recife, PE, Brasil. ORCID: 0000-0002-6708-0450 E-mail: paulosaviogoes@gmail.com

6. Cirurgião Dentista. Mestre e Doutor em Ciências da Saúde. Professor Adjunto da UFPE, Recife, PE, Brasil. ORCID: 0000-0003-3431-115X E-mail: ehglucena@yahoo.com.br
} 


\section{INTRODUÇÃO}

$\mathrm{A}$ proposta de inclusão da saúde bucal no sistema público de saúde ocorreu em 1986, ano da 1a Conferência Nacional de Saúde Bucal (CNSB). No entanto, é a partir de 2003, com o Brasil Sorridente, que criou-se a Política Nacional de Saúde Bucal (PNSB) convergente com os princípios e diretrizes do Sistema Único de Saúde (SUS), o que caracterizou-se por grandes esforços técnicos, com alocação de recursos financeiros sem precedentes, havendo um estímulo para que os sistemas locais buscassem uma nova reorganização do trabalho, contando ainda com a disposição do poder público em organizar a Atenção Básica e ampliar a oferta de procedimentos de média e alta complexidade ${ }^{1}$.

Com a inserção da saúde bucal na Estratégia Saúde da Família (ESF), tornou-se necessário buscar uma nova prática e um olhar diferente sobre o exercício da odontologia no âmbito da saúde coletiva. Esta reorganização da prática odontológica transformou os sujeitos da equipe de saúde bucal, o cirurgião-dentista (CD) e o auxiliar/técnico em saúde bucal (ASB/TSB), em parte de um processo de constante busca pela qualificação profissional, visando integrar um coletivo e desenvolver ações programáticas inseridas nesta estratégia de intervenção populacional baseada em famíliaterritório-comunidade, com vistas à efetivação dos princípios do SUS ${ }^{1}$.

Assim, como uma nova perspectiva de avanço estratégico das ações e dos serviços de saúde pública no Brasil, com foco na superação do desacordo evolutivo entre os Estados, é editado e publicado pelo Ministério da Saúde, no ano de 2006, o Pacto pela Saúde, que visa a consolidação do SUS e aprova as diretrizes operacionais inerentes ao Sistema ${ }^{1,2}$.

0 objetivo passa a ser de promover inovações nos processos e instrumentos de gestão, visando alcançar maior eficiência e qualidade das respostas do SUS, além de explicitar o compromisso entre os gestores de saúde em torno de ações que apresentem impacto sobre a situação de saúde da população brasileira. Ainda de acordo com sua portaria, o Pacto pela Saúde apresenta a definição de prioridades articuladas e integradas em três dimensões complementares: Pacto pela Vida, Pacto em Defesa do SUS e Pacto de Gestão do SUS1,2.

Especificamente para o campo da Odontologia, o Pacto pela Saúde expressa sua relevância no processo de avaliação e monitoramento de programas e serviços da Atenção Básica, pela inclusão de indicadores de saúde bucal: cobertura populacional estimada das Equipes de Saúde Bucal na Estratégia Saúde da Família, média da ação coletiva de escovação dental supervisionada e proporção de exodontia em relação aos procedimentos clínicos ${ }^{2}$.

Desta forma, como importante subsídio para o processo de organização e planejamento dos serviços públicos no Brasil, esses indicadores revelam-se essenciais para o fortalecimento da saúde bucal na Atenção Básica².

A publicação do Decreto ${ }^{\circ} 7.508$, de 28 de junho de 2011, ao regulamentar aspectos da Lei ${ }^{\circ}{ }^{8} .080$, de 19 de setembro de 1990, no tocante ao planejamento da saúde, à assistência à saúde, à articulação interfederativa e à regionalização, entre outros aspectos, cumpre o seu papel no aprimoramento dos processos e práticas inerentes a um novo ciclo de gestão no SUS ${ }^{3}$.

O Ministério da Saúde, o Conselho Nacional de Secretários de Saúde (Conass) e o Conselho Nacional de Secretarias Municipais de Saúde (Conasems) pactuaram, em 28 de fevereiro de 2013, sete premissas norteadoras para a definição do processo de pactuação de indicadores. Com base nessas premissas, foram definidas as Diretrizes, Objetivos, Metas e Indicadores 2013-2015, com vistas ao fortalecimento do Planejamento Integrado do Sistema Único de Saúde e à implementação do Contrato Organizativo de Ação Pública da Saúde (COAP) ${ }^{3}$.

Os indicadores são essenciais nos processos de monitoramento e avaliação, pois permitem acompanhar o alcance das metas e servem para: embasar a análise crítica dos resultados obtidos e auxiliar no processo de tomada de decisão; contribuir para a melhoria contínua dos processos organizacionais; e 
analisar comparativamente o desempenho ${ }^{3}$. Fatores de risco e de proteção incidem de modo desigual sobre os estratos sociais, com efeitos deletérios ou salutares que atingem a população de modo heterogêneo e aumentam as desigualdades em saúde. Nesse sentido, é necessário avaliar as políticas de saúde não apenas pelo efeito global que exercem sobre a saúde coletiva, mas também pelo resultado de suas intervenções sobre o quadro pré-existente de desigualdades em saúde ${ }^{4}$.

Este estudo tem como objetivo analisar o indicador de exodontia em relação às variáveis de contexto dos municípios.

\section{MÉTODO}

Realizou-se um estudo transversal e ecológico. Por meio do DATASUS, coletou-se dados do indicador de produção odontológica "Razão do Número de Exodontias sobre Procedimentos Odontológicos Básicos Individuais" (EXO/POI), referentes ao ano de 2016, de todos municípios brasileiros.

Esse indicador foi dividido em 6 categorias de análise, como a seguir: 0,1\% $5 \%$ (quer dizer que do total de procedimentos clínicos odontológicos básicos individuais realizados no município, de $0,1 \%$ a $5 \%$ foram extrações de dentes permanentes); 5,1\% $12 \%$ (quer dizer que do total de procedimentos clínicos odontológicos básicos individuais realizados no município, entre $5,1 \%$ a $12 \%$ foram extrações de dentes permanentes); $12,1 \%$ - 25\% (quer dizer que do total de procedimentos clínicos odontológicos básicos individuais realizados no município, entre $12,1 \%$ a $25 \%$ foram extrações de dentes permanentes); $25,1 \%$ $50 \%$ (quer dizer que do total de procedimentos clínicos odontológicos básicos individuais realizados no município, entre $25,1 \%$ a $50 \%$ foram extrações de dentes permanentes); $>50 \%$ (quer dizer que do total de procedimentos clínicos odontológicos básicos individuais realizados no município, mais de 50\% foram extrações de dentes permanentes) e sem informação (quando não foi possível calcular o indicador por falta de informação dos municípios no Sistema de Informação Ambulatorial - SIA).
Para análise do contexto dos municípios, foram utilizados os seguintes indicadores: Região do País (Norte, Nordeste, Centro-Oeste, Sul e Sudeste); Proporção de cobertura populacional estimada de saúde bucal na Estratégia Saúde da Família (ESB/ESF); número de Centro de Especialidades Odontológicas (CEO); Índice de Desenvolvimento Humano Municipal (IDHM) e coeficiente de Gini (ou índice de Gini).

0 indicador EXO/POI foi analisado inicialmente por Região, buscando observar as diferenças entre as regiões do país. Relacionou-se também à proporção de cobertura populacional estimada de saúde bucal na Estratégia Saúde da Família (ESB/ESF) de forma dicotomizada, considerando os grupos menor que $50 \%$ e superior ou igual a 50\%. Ao descrever o número de Centros de Especialidades Odontológicas (CEOs) verificou-se os municípios que não possuíam, os que possuíam um, ou mais de um implantado. Ambas as informações foram retiradas da Sala de Apoio à Gestão Estratégica do Ministério da Saúde (SAGE).

O Índice de Desenvolvimento Humano Municipal (IDHM) composto agrega 3 das mais importantes dimensões do desenvolvimento humano: a oportunidade de viver uma vida longa e saudável, de ter acesso ao conhecimento e ter um padrão de vida que garanta as necessidades básicas, representada pela saúde, educação e renda. 0 índice varia de 0 a 1 . Quanto mais próximo de 1 , maior o desenvolvimento humano. Dividindo-se, neste estudo, ao relacionar-se com o indicador, em municípios com IDHM muito baixo, baixo, médio, alto e muito alto ${ }^{5}$.

O Índice de Gini, que aponta a diferença entre os rendimentos dos mais pobres e dos mais ricos, varia de zero a um (alguns apresentam de zero a cem). 0 valor zero representa a situação de igualdade, ou seja, todos têm a mesma renda. 0 valor um (ou cem) está no extremo oposto, isto é, uma só pessoa detém toda a riqueza. Na prática, o Índice de Gini costuma comparar os $20 \%$ mais pobres com os $20 \%$ mais ricos ${ }^{6}$. Levou-se em consideração a média nacional, havendo 
municípios abaixo da média nacional e outros acima da média nacional.

\section{RESULTADOS}

De acordo com a Tabela 1, as Regiões Norte e Nordeste foram as que apresentaram o maior percentual de municípios, $23,3 \%$ e 14,4\% respectivamente, nos quais mais de $25 \%$ dos procedimentos clínicos odontológicos básicos foram extrações. Enquanto que, nas Regiões Sul e Sudeste, em um alto percentual dos municípios, 70,9\% e 83,9\% respectivamente, as extrações correspondem a menos de $12 \%$ dos procedimentos clínicos.

Tabela 1. Razão do Número de Exodontias sobre Procedimentos Odontológicos Básicos segundo a Região do Brasil. 2016.

\begin{tabular}{|c|c|c|c|c|c|c|c|c|c|c|c|c|c|c|}
\hline \multirow{3}{*}{ REGIÃO } & \multicolumn{14}{|c|}{ Indicador EXO/POI } \\
\hline & \multicolumn{2}{|c|}{$0,1 \%-5 \%$} & \multicolumn{2}{|c|}{$5,1 \%-12 \%$} & \multicolumn{2}{|c|}{$12,1 \%-25 \%$} & \multicolumn{2}{|c|}{$25,1 \%-50 \%$} & \multicolumn{2}{|c|}{$>50 \%$} & \multicolumn{2}{|c|}{999} & \multicolumn{2}{|c|}{ Total } \\
\hline & $\mathrm{N}$ & $\%$ & $\mathrm{~N}$ & $\%$ & $\mathrm{~N}$ & $\%$ & $\mathrm{~N}$ & $\%$ & $\mathrm{~N}$ & $\%$ & $\mathrm{~N}$ & $\%$ & $\mathrm{~N}$ & $\%$ \\
\hline $\begin{array}{c}\text { Centro- } \\
\text { Oeste }\end{array}$ & 105 & 22,5 & 201 & 43,0 & 110 & 23,6 & 26 & 5,6 & 3 & 0,6 & 22 & 4,7 & 467 & 100,0 \\
\hline Norte & 70 & 15,6 & 111 & 24,7 & 137 & 30,4 & 81 & 18,0 & 24 & 5,3 & 27 & 6,0 & 450 & 100,0 \\
\hline Nordeste & 158 & 8,8 & 613 & 34,2 & 668 & 37,2 & 208 & 11,6 & 50 & 2,8 & 97 & 5,4 & 1.794 & 100,0 \\
\hline Sul & 378 & 31,7 & 466 & 39,1 & 234 & 19,6 & 39 & 3,3 & 7 & 0,6 & 67 & 5,6 & 1.191 & 100,0 \\
\hline Sudeste & 638 & 38,2 & 761 & 45,6 & 197 & 11,8 & 27 & 1,6 & 12 & 0,7 & 33 & 2,0 & 1.668 & 100,0 \\
\hline Total & 1.349 & 24,2 & 2.152 & 38,6 & 1.346 & 24,2 & 381 & 6,8 & 96 & 1,7 & 246 & 4,4 & 5.570 & 100,0 \\
\hline
\end{tabular}

A considerar a cobertura estimada de saúde bucal na estratégia saúde da família, dos municípios que realizaram mais de $25 \%$ de extrações em relação ao total de procedimentos clínicos, $11,1 \%$ apresentam cobertura menor do que 50\%. Ao mesmo tempo, $63,6 \%$ deste perfil de cobertura realizaram menos de $12 \%$ de extração em comparação aos demais procedimentos clínicos (Tabela 2).

Tabela 2. Razão do Número de Exodontias sobre Procedimentos Odontológicos Básicos segundo a Proporção de Cobertura Populacional Estimada ESB/ESF. Brasil. 2016.

\begin{tabular}{|c|c|c|c|c|c|c|c|c|c|c|c|c|c|c|}
\hline \multirow{3}{*}{$\begin{array}{c}\text { Proporção de } \\
\text { Cobertura } \\
\text { Populacional Estimada } \\
\text { ESB/ESF }\end{array}$} & \multicolumn{14}{|c|}{ Indicador EXO/POI } \\
\hline & \multicolumn{2}{|c|}{$0,1 \%-5 \%$} & \multicolumn{2}{|c|}{$5,1 \%-12 \%$} & \multicolumn{2}{|c|}{$12,1 \%-25 \%$} & \multicolumn{2}{|c|}{$25,1 \%-50 \%$} & \multicolumn{2}{|c|}{$>50 \%$} & \multicolumn{2}{|c|}{999} & \multicolumn{2}{|c|}{ Total } \\
\hline & $\mathrm{N}$ & $\%$ & $\mathrm{~N}$ & $\%$ & $\mathrm{~N}$ & $\%$ & $\mathrm{~N}$ & $\%$ & $\mathrm{~N}$ & $\%$ & $\mathrm{~N}$ & $\%$ & $\mathrm{~N}$ & $\%$ \\
\hline$<50$ & 366 & 23,6 & 621 & 40,0 & 317 & 20,4 & 128 & 8,2 & 45 & 2,9 & 76 & 4,9 & 1.553 & 100,0 \\
\hline$\geq 50$ & 983 & 24,5 & 1.531 & 38,1 & 1.029 & 25,6 & 253 & 6,3 & 51 & 1,3 & 170 & 4,2 & 4.017 & 100,0 \\
\hline Total & 1.349 & 24,2 & 2.152 & 38,6 & 1.346 & 24,2 & 381 & 6,8 & 96 & 1,7 & 246 & 4,4 & 5.570 & 100,0 \\
\hline
\end{tabular}

Fonte: TABNET - DATASUS

Ao relacionar o número de CEOs com o indicador EXO/POI, observa-se que em aproximadamente $10 \%$ dos municípios sem CEOs, 25\% dos procedimentos clínicos odontológicos correspondem a extrações de dentes permanentes, em contraposto aos municípios que possuem no mínimo um CEO. Entretanto, quanto ao percentual, dos municípios com um ou mais de um CEOs, 92,6\% realizaram menos de $12 \%$ de extrações do total de procedimentos clínicos odontológicos (Tabela 3).

Os municípios com IDHM muito alto apresentaram os menores percentuais de extração $(<12 \%)$. Em contrapartida, entre municípios com IDHM muito baixo, 37,5\% realizam mais de $25 \%$ de extrações dentárias, em relação ao total de procedimentos clínicos, como pode ser visto na Tabela 4. 
Tabela 3. Razão do Número de Exodontias sobre Procedimentos Odontológicos Básicos segundo a quantidade de CEO. Brasil. 2016.

$$
\text { Indicador EXO/POI }
$$

Quantidade de CEO $\quad 0,1 \%-5 \% \quad 5,1 \%-12 \% \quad 12,1 \%-25 \%$ 25,1\%-50\% > 50\% 999 Total

\begin{tabular}{ccccccccccccccc} 
& $\mathrm{N}$ & $\%$ & $\mathrm{~N}$ & $\%$ & $\mathrm{~N}$ & $\%$ & $\mathrm{~N}$ & $\%$ & $\mathrm{~N}$ & $\%$ & $\mathrm{~N}$ & $\%$ & $\mathrm{~N}$ & $\%$ \\
\hline $\begin{array}{c}\text { Municípios que NÃO tem CEO } \\
\text { Municípios que tem UM CEO }\end{array}$ & 241 & 31,1 & 377 & 48,6 & 132 & 17,0 & 17 & 2,2 & 7 & 0,9 & 1 & 0,1 & 775 & 100,0 \\
$\begin{array}{c}\text { Municípios que tem MAIS DE } \\
\text { UM CEO }\end{array}$ & 31 & 38,3 & 44 & 54,3 & 5 & 6,2 & 0 & 0,0 & 1 & 1,2 & 0 & 0,0 & 81 & 100,0 \\
\hline Total & 1.349 & 24,2 & 2.152 & 38,6 & 1.346 & 24,2 & 381 & 6,8 & 96 & 1,7 & 246 & $4,45.570$ & 100,0 \\
\hline
\end{tabular}

Fonte: TABNET - DATASUS.

Tabela 4. Razão do Número de Exodontias sobre Procedimentos Odontológicos Básicos segundo o IDHM. Brasil. 2016.

\begin{tabular}{|c|c|c|c|c|c|c|c|c|c|c|c|c|c|c|}
\hline \multirow{3}{*}{ IDHM } & \multicolumn{14}{|c|}{ Indicador EXO/POI } \\
\hline & \multicolumn{2}{|c|}{$0,1 \%-5 \%$} & \multicolumn{2}{|c|}{$5,1 \%-12 \%$} & \multicolumn{2}{|c|}{$12,1 \%-25 \%$} & \multicolumn{2}{|c|}{$25,1 \%-50 \%$} & \multicolumn{2}{|c|}{$>50 \%$} & \multicolumn{2}{|c|}{999} & \multicolumn{2}{|c|}{ Total } \\
\hline & $\mathrm{N}$ & $\%$ & $\mathrm{~N}$ & $\%$ & $\mathrm{~N}$ & $\%$ & $\mathrm{~N}$ & $\%$ & $\mathrm{~N}$ & $\%$ & $\mathrm{~N}$ & $\%$ & $\mathrm{~N}$ & $\%$ \\
\hline $0-0,499$ & 1 & 3,1 & 5 & 15,6 & 7 & 21,9 & 11 & 34,4 & 1 & 3,1 & 7 & 21,9 & 32 & 100,0 \\
\hline $0,500-0,599$ & 97 & 7,1 & 394 & 28,8 & 523 & 38,3 & 208 & 15,2 & 53 & 3,9 & 92 & 6,7 & 1.367 & 100,0 \\
\hline $0,600-0,699$ & 501 & 22,4 & 896 & 40,1 & 579 & 25,9 & 133 & 6,0 & 28 & 1,3 & 96 & 4,3 & 2.233 & 100,0 \\
\hline $0,700-0,799$ & 721 & 38,2 & 841 & 44,5 & 235 & 12,4 & 29 & 1,5 & 12 & 0,6 & 51 & 2,7 & 1.889 & 100,0 \\
\hline $0,800-1$ & 28 & 63,6 & 15 & 34,1 & 0 & 0,0 & 0 & 0,0 & 1 & 2,3 & 0 & 0,0 & 44 & 100,0 \\
\hline 999 & 1 & 20,0 & 1 & 20,0 & 2 & 40,0 & 0 & 0,0 & 1 & 20,0 & 0 & 0,0 & 5 & 100,0 \\
\hline Total & 1.349 & 24,2 & 2.152 & 38,6 & 1.346 & 24,2 & 381 & 6,8 & 96 & 1,7 & 246 & 4,4 & 5.570 & 100,0 \\
\hline
\end{tabular}

Fonte: TABNET - DATASUS

Nos municípios com índice de GINI maior do que a média nacional, $16,3 \%$ apresentaram os maiores percentuais de exodontias, isto é, > 25\%. Por outro lado, em
63,9\% dos municípios com o índice abaixo da média nacional, as extrações foram menos de $12 \%$ do total de procedimentos odontológicos clínicos individuais (Tabela 5).

Tabela 5. Razão do Número de Exodontias sobre Procedimentos Odontológicos Básicos segundo o Índice GINI. Brasil. 2016.

\begin{tabular}{|c|c|c|c|c|c|c|c|c|c|c|c|c|c|c|}
\hline \multirow{3}{*}{ Índice GINI } & \multicolumn{12}{|c|}{ Indicador EXO/POI } & & \\
\hline & \multicolumn{2}{|c|}{$0,1 \%-5 \%$} & \multicolumn{2}{|c|}{$5,1 \%-12 \%$} & \multicolumn{2}{|c|}{$12,1 \%-25 \%$} & \multicolumn{2}{|c|}{$25,1 \%-50 \%$} & \multicolumn{2}{|c|}{$>50 \%$} & \multicolumn{2}{|c|}{999} & \multicolumn{2}{|c|}{ Total } \\
\hline & $\mathrm{N}$ & $\%$ & $\mathrm{~N}$ & $\%$ & $\mathrm{~N}$ & $\%$ & $\mathrm{~N}$ & $\%$ & $\mathrm{~N}$ & $\%$ & $\mathrm{~N}$ & $\%$ & $\mathrm{~N}$ & $\%$ \\
\hline$<0,6$ & 1.299 & 24,6 & 2.069 & 39,3 & 1.247 & 23,7 & 343 & 6,5 & 85 & 1,6 & 227 & 4,3 & 5.270 & 100,0 \\
\hline$\geq 0,6$ & 49 & 16,6 & 82 & 27,8 & 97 & 32,9 & 38 & 12,9 & 10 & 3,4 & 19 & 6,4 & 295 & 100,0 \\
\hline 999 & 1 & 20,0 & 1 & 20,0 & 2 & 40,0 & 0 & 0,0 & 1 & 20,0 & 0 & 0,0 & 5 & 100,0 \\
\hline Total & 1.349 & 24,2 & 2.152 & 38,6 & 1.346 & 24,2 & 381 & 6,8 & 96 & 1,7 & 246 & 4,4 & 5.570 & 100,0 \\
\hline
\end{tabular}

\section{DISCUSSÃO}

Os estudos ecológicos são considerados apropriados para investigar os efeitos do meio sobre a saúde de uma população, assim como para auxiliar na gestão de políticas públicas ${ }^{7}$.

Os estudos transversais são recomendados quando se deseja estimar a frequência com que um determinado evento de saúde se manifesta em uma população específica, além dos fatores associados com o mesmo $^{8}$. Os sistemas de informação em saúde, possibilitam o levantamento de informações, constituindo-se como um instrumento de avaliação.

A escolha da proporção de exodontias é de grande importância para se avaliar as práticas odontológicas que estão sendo realizadas ${ }^{7}$. 
Neste estudo comparou-se o indicador de EXO/POI entre os municípios das Regiões do Brasil, observando a existência de diferenças entre as mesmas. Sendo a situação mais crítica a do Norte, onde aproximadamente $1 / 4$ dos municípios realizaram mais de $25 \%$ de extração dentária, em detrimentos dos demais procedimentos clínicos.

Um estudo ${ }^{9}$ revelou que nos municípios do Estado do Pará, no período 2001-2010, a razão entre o número de exodontias de dentes permanentes e procedimentos odontológicos individuais apresentou redução, apesar de o número de procedimentos anuais ter permanecido estável no período. Isto indica que houve uma mudança ao longo dos anos, tendo diminuído o número de exodontias de dentes permanentes. No entanto, os valores continuam elevados quando comparados às demais localidades.

A cobertura de ESB foi utilizada para comparar a oferta de serviço com o indicador. Embora a Portaria 1.101, de 12 de junho de 2002, do Ministério da Saúde (que estabelece parâmetros assistenciais do SUS) sugira que a relação cirurgião-dentista por habitante pode variar de 1:1.500 a 1:5.000, não há um número ideal estabelecido para essa relação, pois, para determinar tal proporção, é preciso levar em consideração elementos envolvidos no planejamento de recursos humanos odontológicos necessários em cada comunidade $^{7}$. Nos resultados do presente estudo, nos locais de menor cobertura ESB/ESF, o indicador teve uma porcentagem maior para as categorias maiores $(25,1 \%$ $50 \%$ e $>50 \%$ ).

Outro estudo ${ }^{10}$, que analisou a associação entre a cobertura populacional de ESB na ESF e a diferença nas taxas de uso de serviços odontológicos públicos nos municípios brasileiros entre 1999 e 2011, mostrou que o aumento no uso dos serviços odontológicos públicos no Brasil está associado ao aumento nas taxas de ESB da ESF, independente do crescimento da estrutura.

0 aumento da cobertura da assistência individual pode estar privilegiando outros tipos de procedimento, podendo gerar mudanças nas condições de saúde bucal da população que está sendo atendida no serviço público ${ }^{7}$. Apesar do resultado apresentado neste trabalho, pode-se supor que com o tempo esse indicador tende a diminuir nas regiões com maior cobertura.

Determinantes da condição de vida (IDHM) e de saúde (oferta da atenção primária e secundária) das pessoas interferem nas perdas dentárias ${ }^{11}$.

Uma pesquisa ${ }^{12}$ avaliou os CEO em funcionamento no Estado de Pernambuco, observando que quanto maior o IDHM, melhor o desempenho dos Centros de Especialidades Odontológicas.

A oferta da atenção secundária por meio do CEO aumenta o número de procedimentos especializados, mostrando-se essencial para que a rede de serviço de saúde bucal seja menos mutiladora ${ }^{11}$. Os resultados deste estudo corroboram com essa expectativa onde quase $95 \%$ dos municípios, com no mínimo um CEO, realizaram os menores percentuais de extração.

Com relação aos municípios com baixo IDHM, infere-se que, possivelmente, variáveis educacionais e/ou relacionadas à renda devam interferir no acúmulo das necessidades odontológicas da população ${ }^{13}$. Nesse sentido, os municípios com IDHM muito baixo $(<0,499)$ apresentaram os maiores percentuais de extração. A alta proporção de exodontias está em oposição à oferta de procedimentos preventivos e diretamente relacionada às condições socioeconômicas da população ${ }^{14}$.

Noutro estudo ${ }^{15}$, demonstrou-se por meio da regressão beta que a mutilação dentária foi maior nos municípios paraibanos que possuíam valores inferiores ao Índice de Gini, menor número de ESB e menor proporção de procedimentos odontológicos básicos. Enquanto que neste estudo, o percentual de extração maior do que $25 \%$ esteve localizado em municípios com maior concentração de renda, isto é, índice GINI $\geq$ 0,6086 .

A desinformação e, mais ainda, a não conscientização da importância da saúde bucal pela população com menor nível 
educacional têm impacto na condição de saúde bucal, tanto quanto na saúde geral das pessoas $^{13}$.

Espera-se que mais recursos sejam destinados às equipes e que os municípios ampliem sua rede de atenção primária, impactando positivamente na qualidade da saúde bucal da população. Para isso, é importante que haja adoção de políticas públicas de desenvolvimento social que ultrapassem o setor saúde, direcionando o benefício preferencialmente para as áreas onde haja maior necessidade, de forma a contribuir para a redução das desigualdades ${ }^{7}$.

A demora no aperfeiçoamento das informações contidas na base de dados alimentada pelo SUS e disponível para consulta dos pesquisadores também é um problema anunciado.

Espera-se então que os gestores responsáveis pela avaliação e acompanhamento da gestão do SUS estejam mais embasados e cumpram com suas competências.

Cabe aos usuários e aos profissionais exercerem o controle social e reivindicarem o cumprimento dessa avaliação tão importante para o planejamento de melhorias nos serviços de saúde bucal do SUS ${ }^{16}$.

\section{CONCLUSÃo}

Uma das limitações do presente estudo é a utilização de dados secundários, o que significa que os registros podem possuir consistência questionável. Mesmo assim, vale salientar a importância da disponibilidade pública destes dados e sua utilização por pesquisadores, profissionais da saúde e gestores, a fim de ajudar no processo de planejamento e programação das ações e serviços de saúde.

Os resultados do indicador EXO/POI mostram a necessidade de se inverter a oferta dos procedimentos odontológicos e garantir para a população, principalmente a menos favorecida socioeconomicamente, atendimentos e serviços que realizem ações de prevenção, manutenção e recuperação da saúde bucal, e assim revertam a perda dentária.

Entretanto, se fazem necessários novos estudos que avaliem a qualidade dos serviços prestados para que esta não se limite a avaliações quantitativas.

\section{REFERÊNCIAS}

1. Nascimento GE. Avaliação da qualidade dos serviços odontológicos prestados em unidades de saúde da família. 2016. $75 f$. [Dissertação]. Ribeirão Preto, SP: Faculdade de Medicina de Ribeirão Preto, Universidade de São Paulo; 2016. 77 f.

2. Bordin D, Fadel CB. Pacto pela saúde no Brasil: uma análise descritiva da progressão dos indicadores de saúde bucal. Rev Odontol UNESP. 2012; 41(5):305-11.

3. Ministério da Saúde (Br). Caderno de diretrizes: objetivos, metas e indicadores 2013-2015. 3ed. Brasília: Ministério da Saúde; 2015.

4. Antunes JLF, Narvai PC. Políticas de saúde bucal no Brasil e seu impacto sobre as desigualdades em saúde. Rev Saúde Pública. 2010; 44(2):360-5.

5. PROGRAMA DAS NAÇÕES UNIDAS PARA 0 DESENVOLVIMENTO. O que é IDHM [Internet]. Brasília, DF; 2017 [citado em 12 jul 2017]. Disponível em: http://www.br.undp.org/content/brazil/pt/ home/idh0/conceitos/o-que-e-o-idhm.html.

6. Wolffenbüttel A. O que é? - Índice de Gini. Desafios do Desenvolvimento [Internet]. 2004 [citado em 17 jul 2017]; 1(4). Disponível em: http://www.ipea.gov.br/desafios/index.php? option=com_content\&id=2048: catid=28\&Ite mid $=23$.

7. Palmier AC, Andrade DA, Campos ACV, Abreu MHNG, Ferreira EF. Indicadores socioeconômicos e serviços odontológicos em uma região brasileira desfavorecida. Rev Panam Salud Publica. 2012; 32(1):22-9.

8. Bastos JLD, Duquia RP. Um dos delineamentos mais empregados em epidemiologia: estudo transversal. Sci Med. (Porto Alegre). 2007; 17(4):229-32.

9. Silva LS, Santana KR, Pinheiro HHC, Nascimento LS. Indicadores de atenção básica e especializada em saúde bucal nos municípios do Estado do Pará, Brasil: estudo ecológico, 2001-2010. Epidemiol Serv Saúde. 2013; 22(2):325-34.

10. Corrêa GT, Celeste RK. Associação entre a 
cobertura de equipes de saúde bucal na saúde da família e o aumento na produção ambulatorial dos municípios brasileiros, 1999 e 2011. Cad Saúde Pública. 2015; 31(12):2588-98.

11. Cunha, MAGM. Tendência histórica das exodontias de dentes permanentes no Brasil: análise de uma série de 15 anos. [Dissertação]. Belo Horizonte: Faculdade de Odontologia, Universidade Federal de Minas Gerais; 2015. $58 \mathrm{f}$.

12. Figueiredo N, Goes PSA. Construção da atenção secundária em saúde bucal: um estudo sobre os Centros de Especialidades Odontológicas em Pernambuco, Brasil. Cad Saúde Pública. 2009; 25(2):259-67.

13. Cortellazzi KL, Balbino EC, Guerra LM, Vasquez FL, Bulgareli JV, Ambrosano GMB, et al. Variáveis associadas ao desempenho de Centros de Especialidades Odontológicas no Brasil. Rev Bras Epidemiol. 2014; 17(4):97888.

14. Cavalcanti YW, Lira Júnior R, Cartaxo RO, Lucena EHG, Padilha WWN. Associação de indicadores de produção odontológica e de condição sócio-sanitária na atenção básica de João Pessoa-PB. Rev Bras Ciênc Saúde. 2010; 14(3):47-52.

15. Medeiros JJ, Rodrigues LV, Lima Neto EA, Valença AMG, Machado LS. Regressão beta aplicada à avaliação dos indicadores de saúde bucal na Atenção Básica. In: Congresso de Matemática Aplicada e Computacional [Internet]; 2012; Natal, RN. Natal: SBMAC; 2012 [citado em 25 março 2018]. p. 569-572. Disponível em: http://www.sbmac.org.br/cmacs/cmacne/2012/trabalhos/PDF/73.pdf.

16. Lopes, RSVPM. Centros de especialidades odontológicas(CEO) e atenção secundária na saúde bucal: revisão bibliográfica. [Projeto Técnico]. Curitiba: Universidade Federal do Paraná; 2013. 45 f.

\section{CONTRIBUIÇÕES \\ Todos os autores tiveram iguais participações na concepção, delineamento, análise e interpretação dos dados, e redação.}

\section{Como citar este artigo (Vancouver)}

Silva DRB, Lucena CDRX, Cruz DF, Figueiredo N, Goes PSA, Lucena EHG. Análise do indicador de extração dentária a partir do contexto municipal. REFACS [Internet]. 2018 [citado em inserir dia, mês e ano de acesso]; 6(1):220-227. Disponível em: inserir link de acesso. DOI: inserir link do DOI.

\section{Como citar este artigo (ABNT)}

SILVA, D. R. B. et al. Análise do indicador de extração dentária a partir do contexto municipal. REFACS, Uberaba, MG, v. 6, n. 2, p. 220-227, 2018. Disponível em: <inserir link de acesso>. Acesso em: inserir dia, mês e ano de acesso. DOI: inserir link do DOI.

\section{Como citar este artigo (APA)}

Silva, D. R. B.; Lucena, C. D. R. X.; Cruz D. F.; Figueiredo, N.; Goes, P. S. A. \& Lucena, E. H. G. (2018). Análise do indicador de extração dentária a partir do contexto municipal. REFACS, 6(2), 220227. Recuperado em: inserir dia, mês e ano de acesso de inserir link de acesso. DOI: inserir link do DOI. 